\title{
SERVICEABILITY RESPONSE OF A BENCHMARK CABLE-STAYED FOOTBRIDGE: COMPARISON OF AVAILABLE METHODS
}

\author{
Caterina M. RAMOS-MORENO \\ PhD Civil Engineer \\ FAUK \\ London, UK \\ caterina.ramos@ferrovial.com
}

\author{
Ana M. RUIZ-TERAN \\ Senior Lecturer in Bridge \\ Engineering \\ Imperial College London, UK \\ a.ruiz-teran@imperial.ac.uk
}

\author{
Peter J. STAFFORD \\ Reader in Modelling \\ Engineering Risk \\ Imperial College London, UK \\ p.stafford@imperial.ac.uk
}

\begin{abstract}
Summary
In previous Footbridge Conferences, the focus of researchers has been on the representation of pedestrian actions (vertical and lateral) to design footbridges, on the proposal of methodologies for the analysis in service of these structures and on the description of the serviceability response of particular footbridges. Nonetheless, none of these research works have been focused on the magnitude of the serviceability response of footbridges according to its structural type.

This paper characterises the response of footbridges with stayed cables as main structural type. Based on a compiled dataset of cable-stayed footbridges (developed for this research work), the paper presents the geometrical and structural characteristics of a footbridge that can be regarded as representative of this structural type. Considering the best methodologies available for the assessment of its response in vertical and lateral direction, the paper describes the magnitude of the serviceability performance of this bridge under a wide range of pedestrian scenarios. This description familiarises designers at early stages of their design with the order of magnitude of the serviceability response of cable-stayed footbridges with an arrangement similar to that commonly used for this structural type.
\end{abstract}

Keywords: Cable-stayed footbridge; response; vertical acceleration; lateral acceleration; dynamics; serviceability appraisal; benchmark footbridge.

\section{Introduction}

Due to the vast structural knowledge that designers have accumulated during the last decades, engineers and architects have frequently proposed and constructed footbridges with innovative solutions. Some of these have experienced unusually large movements under the action of pedestrian traffic flows and, for this reason, numerous researchers have focused their efforts on the development of better tools to design these structures, in particular the representation of the actions of pedestrians.

In previous Footbridge Conferences, the focus of researchers on pedestrian actions representation is evident as many works presented in these verse on the representation of these for design, the proposal of general methodologies for the analysis in service of footbridges and the serviceability response of specific footbridges.

This paper focuses on an additional tool for designers. This consists in providing designers with a very detailed knowledge of the likely serviceability response of footbridges with a specific structural type: those with stayed cables and geometry similar to those most commonly used by designers when implementing this structural type.

In order to do so, the article describes the geometry of a footbridge that can be considered as representative of this structural type. The geometrical characteristics of this have been extracted from a database (exclusively developed for this research) that includes cable-stayed footbridges designed and constructed until recently. Based on this footbridge, the article describes its serviceability response when using different available 
methodologies and accounting for a wide range of pedestrian events. Considering these responses, the article describes the serviceability comfort that designers can expect to achieve when designing footbridges with this structural type and similar geometry. Additionally, the paper evaluates the available methodologies to assess the structural response of a footbridge with a cable-stayed arrangement or any other.

\section{Previous research}

There is very limited research work focused on the performance in service of cable supported footbridges and in particular for cable-stayed footbridges. For suspension footbridges, there are several research publications that evaluate their performance in service (e.g., Huang et al. [1]). For cable-stayed footbridges there are fewer publications. These are mainly focused on the response of specific cases, Biliszczuk et al. [2], and none have been focused on finding the likely response in service of a footbridge with geometric characteristics that matches those that many designers implement when developing footbridges with this particular structural type. Thus, the aim of this paper is to present some results in this area.

\section{Characteristics of the footbridge representative of the cable-stayed bridge typology}

\subsection{Geometry of the conventional cable-stayed footbridge}

The cable-stayed footbridge considered to be representative of footbridges with this structural type has geometric and material characteristics that have been extracted from the parameters observed in real structures, a compilation of 38 constructed cable-stayed footbridges that have been found in relevant literature (e.g., publications in past Footbridge conferences, in footbridge design books such as that of Baus et al. [3], Strasky [4] or Svensson [5] or published in Structurae [6]). This dataset has been developed for this work and further details can be found in Ramos [7].

According to this compilation, a conventional or stereotypical cable-stayed footbridge has a configuration with main spans that cover distance between 50 and $100 \mathrm{~m}$ (distances around $150 \mathrm{~m}$ are considerably less usual), with a median value being closer to $50 \mathrm{~m}$.

The proposed conventional cable-stayed footbridge has a configuration with the following characteristics:

1. Usually, cable-stayed footbridges have two or three spans, being two the most common arrangement. The proposed conventional footbridge has a main span $\left(\mathrm{Lm}_{\mathrm{m}}\right)$ of length $50 \mathrm{~m}$ with a side span $\left(L_{s}\right)$ of length $0.20 \mathrm{Lm}$.

2. The deck of the footbridge consists of steel girders with a concrete slab. The compiled dataset shows that the most usual arrangements include steel box girders, steel girders with slabs with materials other than concrete and steel girders with concrete slabs. The second one (slightly more popular than the rest) is chosen for the conventional cable-stayed footbridge.

3. The most favoured pylon design has a single vertical mono-pole configuration (transverse cross section is a steel circular hollow section). Considerably less used tower sections correspond to those with shale 'A', 'Y' or 'H'.

4. The database shows that the most used tower elevation has a magnitude near $0.36 \mathrm{Lm}$ (see Fig. 1), magnitude that is considered for the conventional cable-stayed footbridge.

5. The footbridges of the collected dataset describe (see Fig. 1) how for the chosen main span length the deck depth has a value equivalent to $\mathrm{L}_{\mathrm{m}} / 100$ (i.e., $0.5 \mathrm{~m}$ ).

6. The cables are located in a modified fan disposition (fan arrangement with cables at the pylon anchored at different locations), instead of a harp disposition as this is most favoured by designers of these footbridges. In relation to the distance between anchorages of the cables at the deck, the database shows no correlation between this magnitude and the main span length hence its median value $(7 \mathrm{~m})$ is used to choose the number and location of cables of the bridge.

7. The deck width magnitude depends on the size of the pedestrian flows expected to use the footbridge. The footbridges of the compiled dataset have an average deck width of $4 \mathrm{~m}$, value that is adopted for the conventional cable-stayed footbridge. 
8. The footbridge boundary conditions have a large impact on the structural response both in vertical and lateral directions. At the embankments, the arrangement considered consists of laminated elastomeric bearings (dimensions $200 \times 200 \times 32$ ) and a shear key restricting lateral movements of the deck. At the pylon, this has joint displacements with the deck (rotations are not transmitted).
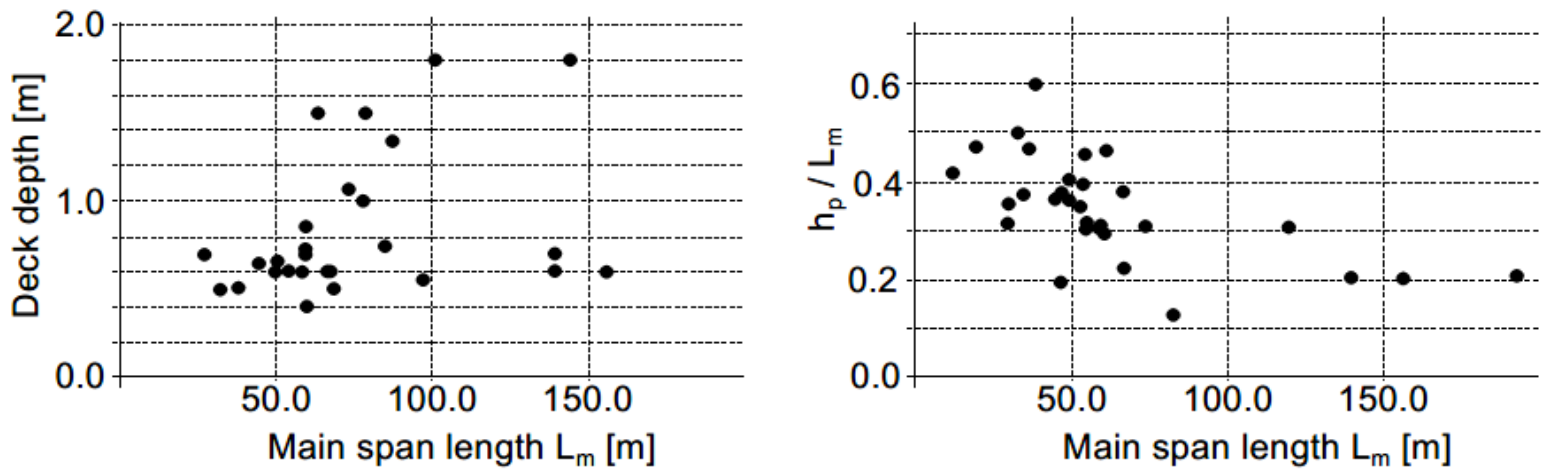

Fig. 1. Deck depth (left) and tower height (right) according to main span length of the cable-stayed bridge.

The particular dimensions of each structural element have been derived considering their performance under permanent and live loads. As permanent loads, their self-weight and that of balustrades. As live loads, those of pedestrian traffic specified by the NA to BS EN 1991-2:2003 [8], wind and temperature (assuming the bridge being located $125 \mathrm{~m}$ above the sea, $7.5 \mathrm{~m}$ above the ground and near London). The geometry of the cablestayed footbridge considered to be representative of this structural type is shown in Fig. 2.

Sec. A-A:

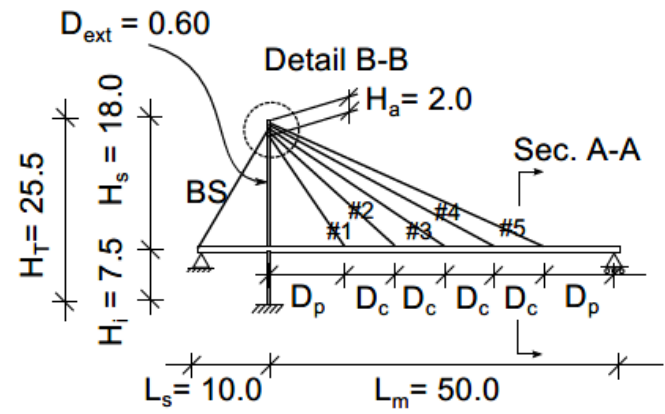

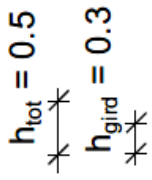

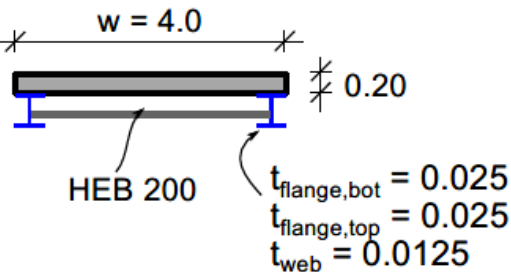

Detail B-B:

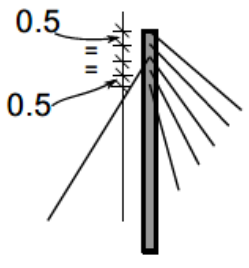

Restricted movements

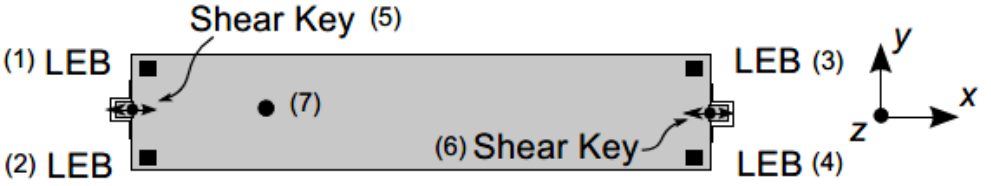
at supports:

\begin{tabular}{|c|c|}
\hline Region & Restrictions \\
\hline$(1)-(4)$ & $\mathrm{Kx}, \mathrm{Kv} U \mathrm{z}$ \\
\hline$(5)-(6)$ & Uy \\
\hline (7) & Ux, UX, Uz(*) \\
\hline
\end{tabular}

Fig. 2. Geometric and structural characteristics of the conventional cable-stayed footbridge; and articulation of the footbridge deck (movements restricted by supports). Dimensions in [m].

\subsection{Fundamental dynamic characteristics of the conventional cable-stayed footbridge}

Design guidelines emphasise that the sensitivity of footbridges to dynamic pedestrian actions is related to the coincidence of the footbridge vibration frequencies to the ranges of frequencies used by individual pedestrians while walking. In lateral direction, this correlation between structure modal frequencies and response is not so straightforward and codes and guidelines highlight the possibility of pedestrians generating large lateral responses even when footbridges have lateral modes well below 1.5 Hz (e.g., the NA to BS EN 1991-2:2003 [8]). 
The first vibration modes of the conventional cable-stayed footbridge are described in Table 1. These have been obtained from the numerical representation of the cable-stayed footbridge through a Finite Element Model developed with ABAQUS, a commercial software widely used in research analyses in the civil engineering field. In this model, the slab is characterised through shell elements, the steel girders with shell elements with thickness according to their dimensions of web and flanges, the transverse steel girders with beam elements and the cables with single truss elements.

This Table 1 includes modes when the structure is empty or subjected to the passage of different pedestrian flow densities (some guidelines suggest including the mass of the pedestrian flow to describe the structural frequencies, e.g., Zivanovic et al. [9]).

The modes described in Table 1 show how moderate span length cable-stayed footbridges with composite decks have vertical modes within the range considered critical whereas for lateral or torsional movements frequencies are beyond the commonly assumed critical ranges.

\begin{tabular}{cccccc}
\hline Mode Number & $\begin{array}{c}\text { Empty } \\
\text { footbridge }\end{array}$ & $\mathbf{0 . 2} \mathbf{~ p e d} / \mathbf{m}^{\mathbf{2}}$ & $\mathbf{0 . 6} \mathbf{~ p e d} / \mathbf{m}^{\mathbf{2}}$ & $\mathbf{1 . 0} \mathbf{~ p e d} / \mathbf{m}^{\mathbf{2}}$ & $\begin{array}{c}\text { Mode } \\
\text { description }\end{array}$ \\
\hline 1 & 1.01 & 1.00 & 0.98 & 0.96 & $\mathrm{~V} 1$ \\
2 & 1.14 & 1.13 & 1.11 & 1.09 & $\mathrm{Ld}$. \\
3 & 1.21 & 1.20 & 1.20 & 1.20 & $\mathrm{P}$ \\
4 & 2.02 & 2.00 & 1.96 & 1.92 & $\mathrm{~V} 2$ \\
5 & 2.23 & 2.21 & 2.16 & 2.12 & $\mathrm{~L} 1$ \\
6 & 2.98 & 2.96 & 2.93 & 2.89 & $\mathrm{~T} 1$ \\
7 & 3.28 & 3.25 & 3.18 & 3.12 & $\mathrm{~T} 2$ \\
8 & 3.82 & 3.79 & 3.73 & 3.67 & $\mathrm{~V} 4$ \\
9 & 5.08 & 5.03 & 4.92 & 4.83 & $\mathrm{~T} 3$ \\
10 & 5.57 & 5.52 & 5.44 & 5.36 & $\mathrm{P}$ \\
11 & 7.02 & 7.01 & 6.99 & 6.96 & $\mathrm{~V} 5$ \\
12 & 7.44 & 7.36 & 7.20 & 7.06 & \\
\hline
\end{tabular}

Table 1. Vibration modes and frequencies [Hz] of the conventional cable-stayed footbridge, where ' $V N$ ', 'TN' and ' $L N$ ' denote vertical, torsional and lateral modes with $N$ half-waves in the main structural span (i.e., from the pylon to the abutment support section, for vertical and torsional modes, and between abutment support sections for lateral modes), 'Ld' longitudinal and ' $P$ ' pylon modes.

\section{Methodologies for the assessment of the serviceability response of the conventional cable- stayed footbridge}

A satisfactory prediction of the response of footbridges in service relies upon the assumptions made to represent the human actions and the pedestrian scenarios. However, anthropogenic loads and scenarios have a significant degree of variability due to: the wide range of anthropometric characteristics of human population, the inability of humans to repeat monotonously the same activity and the different constraints that occur in any multiple pedestrian scenario (interactions between subjects).

In relation to vertical loads, since the first formulation published in 1977, multiple research groups have focused their attention to the reformulation of human actions in order to take into account their intra-variability, intervariability and collective behaviour (e.g., Zivanovic et al. [10]). For lateral loads, efforts started slightly later, mainly after the event recorded at the Millennium footbridge in London.

Some load models that are currently used for footbridge design have considered some of these issues as it is the case of the frequently used proposals of the NA to BS EN 1991-2:2003 [8], the Setra Guideline [11] or the method proposed by Georgakis et al. [12]. In lateral direction, there are considerably fewer methods to predict the serviceability response. A method that is widely known corresponds to that proposed by Setra [11]. Alternatively, the UK National Annex [8] provides an assessment to appraise the possibility of the bridge developing unstable lateral response. Nonetheless, this second alternative poses a question in relation to the possibility of a footbridge deemed by that proposal as adequate developing large lateral movements (providing minimum comfort to users) without these being unstable. 
As an alternative to these methods, the authors of this article have recently proposed a novel pedestrian load model that is capable of representing the vertical and lateral pedestrian loads including the three components of their variability (intra-subject variability, inter-subject variability and collective behaviour) in a realistic manner. The model is based on experimental data and contrasted with experimental recording (for vertical loads this is founded on an empirical dataset described in Butz et al. [13], for lateral loads on the work of Townsend [14], Macdonald [15] and Carroll et al. [16] and pedestrian traffic condition on the work developed by Weidmann [17] among others). The model describes the temporal evolution of load amplitudes (vertical and lateral, see Fig. 3) associated with individual steps as functions of the gait characteristics (pedestrian speed and step frequency) and of the subject properties (e.g., pedestrian mass, age and height). This load description is used to represent the loads transmitted in events with multiple pedestrians that may interact by avoiding one another or walking in groups by changing their direction of movement, speed, step length and step frequency. This proposal has been developed from a meta-analysis of the state-of-the-art multidisciplinary research related to these topics that has been recently published (further details can be found in Ramos [7]).
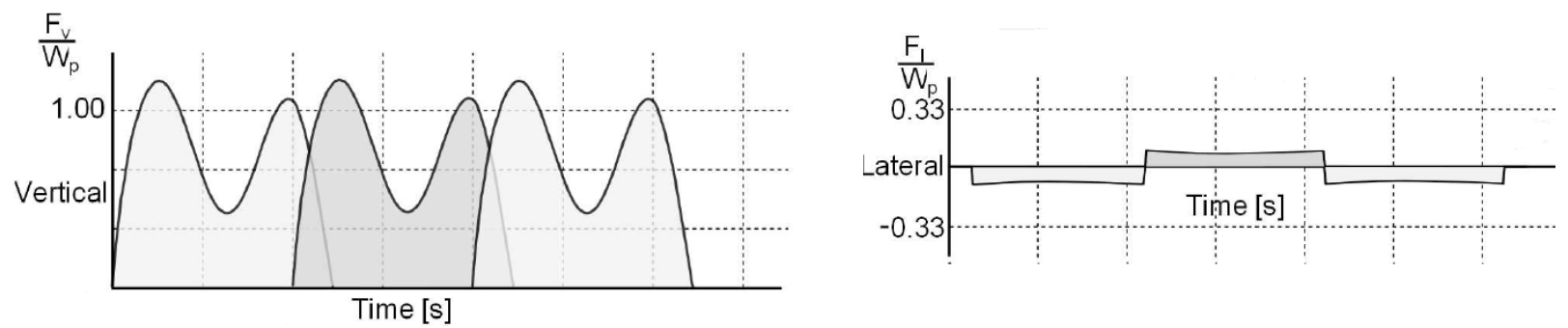

Fig. 3: Definition of consecutive vertical and lateral pedestrian loads according to the novel pedestrian load model [3].

\section{Characteristics of the pedestrian traffic}

Design guidelines highlight that the sensitivity of a footbridge to the effects of a pedestrian flow is influenced by the step frequencies adopted by pedestrians when crossing the structure.

The approach of most of the methods stated in Section 4 consists in defining the characteristics of the worst case scenario for the bridge depending on the coincidence of the structure modal frequencies and higher harmonics with the most common human step frequencies.

As an alternative to this approach, the load model proposed by the authors focuses on the definition of the pedestrian events regardless the dynamic characteristics of the footbridge. The model relies on being able to describe any possible scenario and designers choosing the most adequate according to the likelihood of each event occurring during the footbridge lifetime. The novel model allows for the description of a pedestrian event according to the number of individuals on the bridge at the same time and the purpose of their journey. Any pedestrian may be crossing the bridge while going to work or school (commuting), travelling for work reasons (business) or strolling for leisure (leisure). This aim and traffic density have an effect on the walking speed, step frequency and step length that each pedestrian uses.

Considering that the conventional cable-stayed footbridge might be located at different settings, this paper presents the serviceability response that this footbridge might develop when being used by pedestrian events with small, medium and large numbers of users and where pedestrians were commuting or strolling. These events correspond to pedestrian traffics with an average number of pedestrians on the bridge deck of $0.2,0.6$ or 1 pedestrian per deck metre squared $\left(\mathrm{ped} / \mathrm{m}^{2}\right.$ ) while commuting or strolling for leisure (walking faster or slower depending on the aim of their journey).

Fig. 4 and 5 describe the initial mean vertical step frequencies used by each pedestrian when entering the bridge in each case. These mean frequencies do not take into account the intra-variability of each human and the fact that users may slow down when encountering others blocking their way.

When comparing the distributions of initial step frequencies of Fig. 4 and 5 against the ranges of most critical frequencies highlighted by the Setra Guideline [11], 1.7-2.1Hz, or the NA to BS EN 1991-2:2003 [8], 1.8-2.0Hz, it is clear that at the events with a light flow of commuters and a heavy traffic of leisure pedestrians mainly use frequencies beyond the critical ranges. 


\section{Serviceability response and comfort appraisal}

According to the novel methodology proposed for the appraisal of footbridge serviceability, the response of the conventional cable-stayed footbridge has magnitudes as described in Fig. 6 . These figures represent the peak accelerations of the events (of events with a time duration sufficient to ensure that the response of the bridge reaches a steady state) at different points along the deck length but also 1s-RMS accelerations. These correspond to values of accelerations weighted with time and are used here in contrast to the peak values to evaluate how often in time accelerations similar to the peak values occur (e.g., Barker et al. [18]).

As shown in Fig. 6 , the maximum vertical response in any scenario occurs at $x=28.0 \mathrm{~m}$ and $50.0 \mathrm{~m}$, which are the antinodes of mode V2. In the lateral direction, the peak response is produced at $x=30.0 \mathrm{~m}$, which is
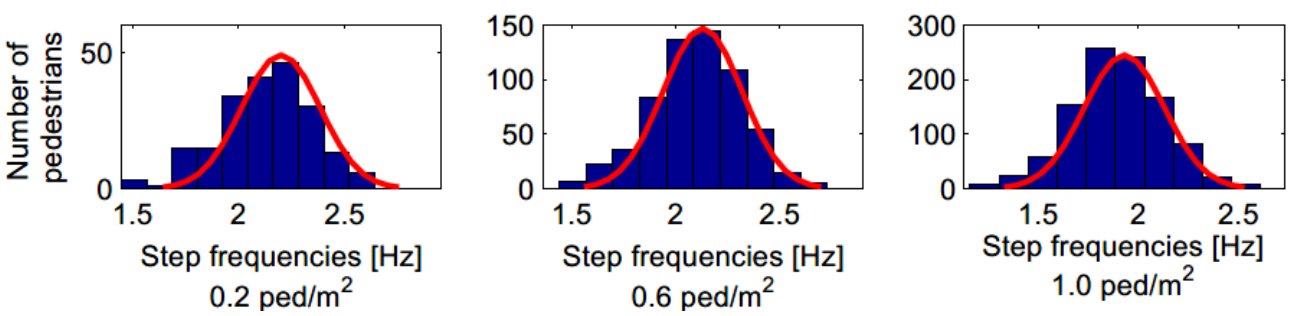

Fig. 4: Distribution of initial mean vertical step frequencies adopted by commuting pedestrians in flows of $0.2,0.6$ and $1.0 \mathrm{ped} / \mathrm{m}^{2}$ (blue bars describe frequencies of the actual event considered in this paper and red lines describe the theoretical normal distribution that characterises these commuting events).
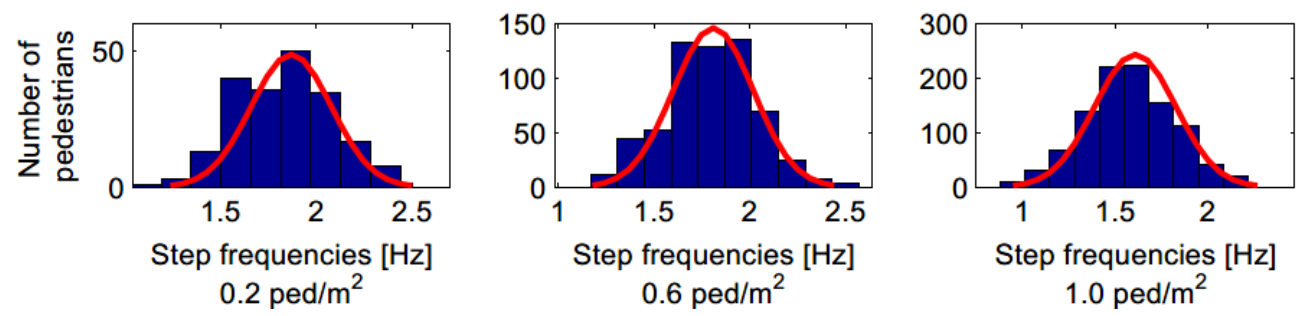

Fig. 5: Distribution of initial mean vertical step frequencies adopted by leisure pedestrians in flows of 0.2, 0.6 and $1.0 \mathrm{ped} / \mathrm{m}^{2}$ (blue bars describe frequencies of the actual event considered in this paper and red lines describe the theoretical normal distribution that characterises these commuting events).

the antinode of the first lateral mode L1. Further analysis of the results obtained with the proposed method show that:

1. In general, the accelerations in vertical and lateral direction are larger when pedestrians are commuting rather than strolling, regardless the number of pedestrians of the event.

2. In the vertical direction, the response is generated by modes V1 and V2 but as well T1 and T2. In the lateral direction, the accelerations have noticeable magnitudes despite the first lateral mode $\mathrm{L} 1$ having a modal frequency around $2.2 \mathrm{~Hz}$ (well beyond $1.5 \mathrm{~Hz}$ )

3. The serviceability response (vertical and lateral) of the events with a small number of commuters and a large number of users while strolling are slightly larger than predicted (if considering that the initial step frequencies described in the previous section are directly related to the expected response) due to the contribution of torsional modes T1 and T2 (in vertical and lateral directions) to the peak and 1sRMS accelerations.

The serviceability responses of this footbridge obtained using other methodologies are given in Table 2:

1. The vertical accelerations described by the NA to BS EN 1991-2:2003 [8] have an order of magnitude similar to that of the proposed model although for medium and large density flows the maximum accelerations are moderately larger.

2. The vertical accelerations reproduced by Setra [11] are considerably larger than those of the rest of models regardless of the traffic flow density. For medium and large density events its results may be unlikely to occur as users would change their behaviour (they would stop or walk in a different manner) 
while sensing such large vibrations. On the other hand, the lateral accelerations predicted by this model are substantially small in comparison to those of the proposed load model. According to these, the bridge would practically remain static despite pedestrians crossing the footbridge.

3. The proposal of Georgakis et al. [12] describes the vertical accelerations that are most similar (although moderately smaller) to those of the proposed load model. The definition of the method allows considering different types of traffic flows, with different aim of journey.

In terms of comfort of users, there are several proposals that assess this according to the magnitude of the serviceability response. Due to the fact that there are multiple parameters influencing the perception of humans, comfort is usually evaluated considering ranges that describe different degrees of comfort (rather than a single boundary describing what is comfortable or not). For footbridges specifically, there are proposals such as that of the British Standard [8], Setra [11] or Synpex [13]. According to these, in vertical direction comfort is maximum, medium or minimum if peak accelerations are between $0-0.5 \mathrm{~m} / \mathrm{s}^{2}, 0.5-1.0 \mathrm{~m} / \mathrm{s}^{2}$ and $1.0-$ 2.0 (or 2.5$) \mathrm{m} / \mathrm{s}^{2}$ respectively. In lateral direction the equivalent ranges are $0-0.1 \mathrm{~m} / \mathrm{s}^{2}, 0.1-0.3 \mathrm{~m} / \mathrm{s}^{2}$ and 0.3 $0.8 \mathrm{~m} / \mathrm{s}^{2}$. Beyond the largest value, comfort is unacceptable for users.
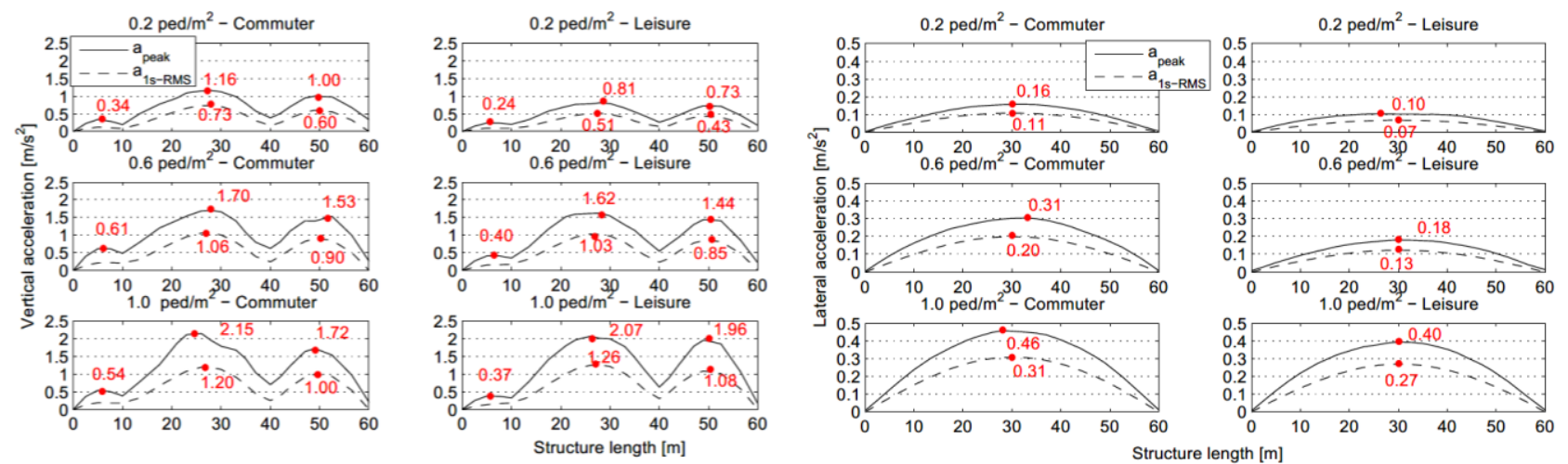

Fig. 6: Peak and 1s-RMS vertical (left) and lateral (right) accelerations recorded at the deck of the conventional cable-stayed footbridge generated by commuter or leisure flows with $0.2,0.6$ and $1.0 \mathrm{ped} / \mathrm{m}^{2}$. The origin for the abscissa axis is located at the support section of the side span on the abutment.

\begin{tabular}{|c|c|c|c|c|c|c|c|}
\hline \multirow[b]{2}{*}{ Method } & \multirow[b]{2}{*}{$\begin{array}{c}0.2 \\
\mathrm{ped} / \mathrm{m}^{2}\end{array}$} & \multirow[b]{2}{*}{$\begin{array}{c}0.6 \\
\mathrm{ped} / \mathrm{m}^{2}\end{array}$} & \multirow[b]{2}{*}{$\begin{array}{c}1.0 \\
\mathrm{ped} / \mathrm{m}^{2}\end{array}$} & \multirow[b]{2}{*}{ Method } & \\
\hline & & & & & $\begin{array}{c}0.2 \\
\mathrm{ped} / \mathrm{m}^{2}\end{array}$ & $\begin{array}{c}0.6 \\
\mathrm{ped} / \mathrm{m}^{2}\end{array}$ & $\begin{array}{c}1.0 \\
\mathrm{ped} / \mathrm{m}^{2}\end{array}$ \\
\hline $\begin{array}{c}\text { Proposed } \\
\text { method }\end{array}$ & $\begin{array}{c}1.16(\mathrm{C}) / \\
0.81(\mathrm{~L})\end{array}$ & $\begin{array}{c}1.70(\mathrm{C}) / \\
1.62(\mathrm{~L})\end{array}$ & $\begin{array}{c}2.15(\mathrm{C}) / \\
2.07(\mathrm{~L})\end{array}$ & $\begin{array}{l}\text { Proposed } \\
\text { method }\end{array}$ & $\begin{array}{c}0.16(\mathrm{C}) / \\
0.10(\mathrm{~L})\end{array}$ & $\begin{array}{c}0.31(\mathrm{C}) / \\
0.18(\mathrm{~L})\end{array}$ & $\begin{array}{c}0.46(\mathrm{C}) / \\
0.40(\mathrm{~L})\end{array}$ \\
\hline$N A$ to $B S$ & 1.13 & 1.99 & 2.47 & SETRA & 0.01 & 0.02 & 0.08 \\
\hline
\end{tabular}

EN 1991-

2:2003

SETRA

Georgakis

1.89

3.21

11.89

et al.

$0.90(\mathrm{C}) / \quad 1.56(\mathrm{C}) / 2.01(\mathrm{C}) /$

$\begin{array}{lll}0.83(\mathrm{~L}) & 1.44(\mathrm{~L}) \quad 1.86(\mathrm{~L})\end{array}$

Table 2. Comparison of the vertical (left) and lateral (right) performance of the conventional cable-stayed footbridge estimated by the proposed method and available alternative proposals.

According to the comfort ranges previously stated, in the vertical direction the comfort of the footbridge is equivalent to minimum (medium for small flows of leisure pedestrians) and unacceptable for large flows of pedestrians. In the lateral direction, the comfort decreases from medium to minimum with increasing pedestrian density (except for small density of users while strolling where comfort is maximum).

If instead of the peak accelerations, the 1s-RMS are taken into account to assess the accelerations of the footbridge, the comfort of this conventional cable-stayed footbridge is slightly better: minimum in vertical direction (except for small flows where this is medium) and to maximum or medium in lateral direction.

Despite the fact that peak accelerations are currently used to assess the degree of comfort of footbridges, results show that others such as RMS accelerations might be more realistic. Fig. 7 depicts the area and time of the event (scenarios with $0.6 \mathrm{ped} / \mathrm{m}^{2}$ of commuters) where vertical and lateral accelerations are equal or 
larger than different values. These figures clearly show that only during less than $10 \%$ of the time and area of the deck accelerations are similar to the absolute peak values and thus only a small proportion of users is likely to sense this peak movement (vertical or lateral). Hence, judging the comfort of a footbridge based on peak values may be far too pessimistic.
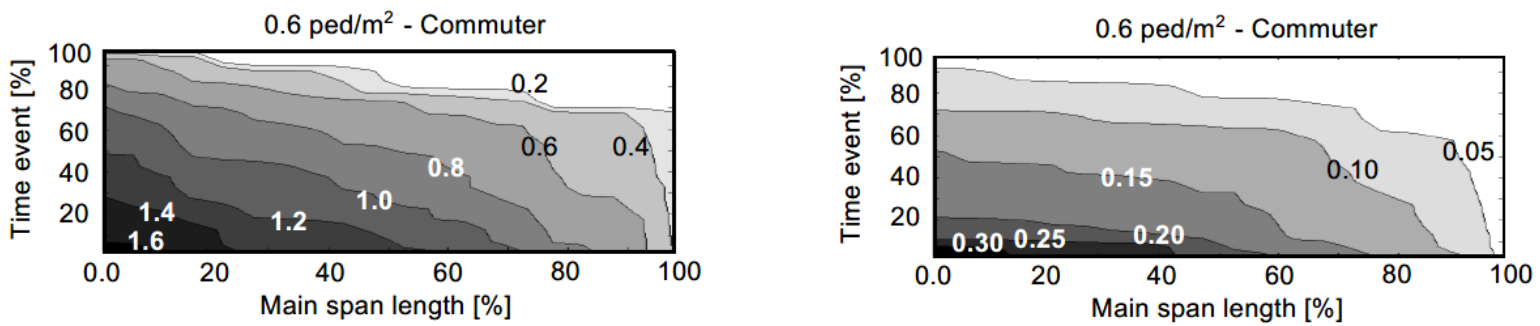

Fig. 7: Percentage of time and main span surface for which the maximum vertical (left) and lateral (right) accelerations recorded at the deck are larger than the value indicated in the contour curves.

\section{Discussion and Conclusions}

The results described in this paper show that the level of comfort of the serviceability response of the conventional cable-stayed footbridge (with a moderate main span length of $50 \mathrm{~m}$ ) is equivalent to minimum/unacceptable or medium/minimum in vertical or lateral direction respectively. If instead of peak accelerations the 1s-RMS accelerations are taken into account, the comfort of this footbridge is usually equivalent to minimum and medium/minimum in vertical or lateral direction respectively. In the vertical direction, the comfort levels are practically the same when using methods such as that proposed by the authors, the UK National Annex [8] or Georgakis et al. [12]. In the lateral direction, exclusively the proposed method provides a reliable assessment. If other values (instead of peak or 1s-RMS accelerations) were used to assess the comfort of this footbridge (such as the maximum acceleration recorded during a \% of the time and area of the footbridge deck) a considerably better comfort assessment would be obtained.

In relation to any footbridge in general, the vertical serviceability response predicted by the methods of the UK National Annex [8], Georgakis et al. [12] or the new proposal of the authors of this paper are fairly similar although very realistic and accurate values can be obtained by using the new methodology. In lateral direction there are currently no available methods except for that proposed by the authors of this article (Setra [11] provides unrealistic responses). Hence, in order to grasp the footbridge lateral response in a realistic manner, the model proposed by the authors is the most adequate method currently available.

\section{Acknowledgements}

The authors are grateful for the support received by the first author from "La Caixa" Foundation to fund her $\mathrm{PhD}$ studies at Imperial College London.

\section{References}

[1] HUANG M.-H., THAMBIRATHAM D.P., and PERERA N.J., "Resonant Vibration of Shallow Suspension Footbridges", Proceedings of ICE, Bridge Engineering 15 (1), 2005, pp. 201-209.

[2] BILISZCZUK J., BARCIK W., HAWRYSZKOW P. et al., "Dynamic Sensibility of Cable-Stayed Footbridges", Proceedings of Footbridge 2005, Second International Conference, 2005.

[3] BAUS U. and SCHLAICH M., "Footbridges: Construction, Design, History”, Birkhäuser, 2007.

[4] STRASKY J., "Stress ribbon and cable-supported pedestrian bridges", Thomas Telford, 2005.

[5] SVENSSON H., "Cable-stayed bridges. 40 years of experience worldwide", Ernst \& Sohn, 2012.

[6] STRUCTURAE, "Structurae: International database for civil and structural engineers", URL: http://structurae.net.

[7] RAMOS-MORENO C., "Design of Cable-Stayed Footbridges under Serviceability Loads", PhD Thesis Imperial College London, 2016. 
[8] BSI (2008). "NA to BS EN 1991-2:2003. UK National Annex to Eurocode 1: Actions on Structures. Part 2: Traffic loads on bridges". BS. London, UK.

[9] ZIVANOVIC S., PAVIC A. and INGOLFSSON E.T., "Modeling Spatially Unrestricted Pedestrian Traffic on Footbridges", Journal of Structural Engineering - ASCE 136, 2010, pp. 1296-1308.

[10] ZIVANOVIC S., PAVIC A. and REYNOLDS P., "Probability Based Prediction of Multi-Mode Vibration Response to Walking Excitation”, Engineering Structures 29 (6), 2007, pp. 942-954.

[11] CHARLES P., HOORPAH W., BONIFACE V. et al., "Footbridges. Assessment of Vibrational Behaviour of Footbridges under Pedestrian Loading. Guideline", 2006, SETRA/AFGC.

[12] GEORGAKIS C.T. and INGOLFSSON E.T. "Vertical Footbridge Vibrations: the Response Spectrum Methodology", Footbridge 2008, Third International Conference, 2008.

[13] BUTZ C., FELDMANN M., HEINEMEYER C. et al. "Advanced Load Models for Synchronous Pedestrian Excitation and Optimised Design Guidelines for Steel Footbridges", Synpex. European Commission. Research Fund for Coal and Steel, 2008.

[14] TOWNSEND M., "Biped gait stabilization via foot placement", Journal of Biomechanics 18, 1985, pp. 21-38.

[15] MACDONALD J.H.G., "Lateral excitation of bridges by balancing pedestrians", Proceedings of Royal Society A-Mathematical Physical and Engineering Sciences 465, 2009, pp. 1055-1073.

[16] CARROLL S.P., OWEN J.S. and HUSSEIN M.F.M, "Modelling crowd-bridge dynamic interaction with a discretely defined crowd", Journal of Sound and Vibration 331 (11), 2012, pp. 2685-2709.

[17] WEIDMANN U., "Transporttechnik der Fussgaenger - Transporttechnische Eigenshaften des Fussgaengerverkehrs. Literaturstudie", Institut für Verkhersplanung, Transporttechnik, Strassen un Eisenbahnbau, IVT an der ETH Zurich.

[18] BARKER C., DENEUMANN S., MACKENZIE D. and KO R., "Footbridge Pedestrian Vibration Limits. Part 1: Pedestrian Input", Footbridge 2005, Second International Conference, 2005. 